\title{
"Reverse axillary mapping in early stage breast cancer Is it oncologically safe? A feasibity study"
}

\author{
${ }^{1}$ Dr. Balasubramanian V ,MCh, ${ }^{2}$ Dr. Sridevi V, ${ }^{3}$ Dr. Seenu Vathulru, \\ ${ }^{4}$ Dr. Asish Jakhetiya \\ ${ }^{1,2}$ Department of Surgical Oncology, Cancer Institute (WIA), Chennai \\ ${ }^{3}$ Professor, Chief Unit 4,Department of Surgical Disciplines, All India Institute of Medical Sciences, AIIMS, New \\ Delhi \\ ${ }^{5}$ Assistant professor, Department of surgical Oncology Vardhaman Mahavir Hospital, New Delhi
}

\begin{abstract}
Background: Arm lymphatics draining through the axilla are frequently disrupted during surgery for carcinoma breast leading to lymphedema. Techniques for differential identification of arm lymphatics might enable their preservation, and probable reduction in lymphedema.

Aims: To evaluate the efficacy and oncological safety of reverse axillary mapping (RAM) in patients with early stage breast carcinoma and to correlate with post operative pathological nodal status.

Methods and Material: It is a prospective pilot study, conducted at a tertiary care cancer speciality hospital, among patients with early stage breast cancer ( $c T 1 / T 2$ cN1M0) planned for straight surgery, consenting to be part of the study. Patients were subjected to injection of blue dye (total $1 \mathrm{ml}$ ) at the time of surgery. During axillary dissection, lymphnodes taking up blue dye, were identified, dissected out and sent separately for pathological assessment .

Results: RAM technique with blue dye identified arm lymphatic in ipsilateral axilla in $72.22 \%$. Blue lymph nodes were identified in $61.11 \%$. Postoperatively 13 out of 18 patients were staged as $p N O$, two as $p N 1$, and three as pN3. RAM identified arm lymph nodes were free of diseases in all the patients with pNO,pN1 patients, only one of the three pN3 patients showed metastatic involvement . ( all pN3 patients could be identified as suspicious of having high nodal disease intraoperatively)
\end{abstract}

Conclusions: Reverse axillary mapping can be used to identify the arm lymphatics in the ipsilateral axilla and these nodes are found to be free of metastatic disease in early stage breast cancer.

\section{Background}

Lymphatic drainage of the breast and ipsilateral upper limb is through the axilla. Majority of the cases, the two lymphatic pathways are different[1] Technique, that can differentially identify the upper limb lymphatic in the axilla can help preventing disruption of arm lymphatics at the time of axillary dissection for breast cancer. This may help reduce lymphedema, the most significant morbidity associated with surgical management of breast cancer. Reverse axillary mapping (RAM) technique, is a recent development, aimed at identifying the arm lymphatics in axilla at the time of axillary surgery for carcinoma breast. Various techniques have been described for the same - using blue dye ,radioisotope or fluorescence imaging [2-5]. Although arm and breast lymphatics have been postulated to have differential drainage crossover has been documented in 2-18\% [1,5-7] with pathologically proven involvement of nodes draining the arm by metastatic carcinoma from breast in 0 $43 \%[5,7-11]$. Most common complication associated with this procedure has been local tattoing lasting from few weeks to as long as 6 months. We aim to evaluate the efficacy of identification of arm lymphatic using low volume methylene blue injection $(1-1.5 \mathrm{ml})$ and the degree of involvement of RAM identified nodes by metastatic disease among women with early stage breast cancer.

\section{Methods}

The study was conducted as a prospective non randomised study. It was conducted in the Division of breast oncology, Department of Surgical oncology at our tertiary care centre.

\section{Subjects}

Female patients with early stage breast cancer (cT1/T2) satisfying the exclusion criteria, with soft clinically palpable node $(\mathrm{cN} 1)$ after routine metastatic work up , consenting to be a part of study were included in the study. Written informed consent, explaining the procedure and possible complications were taken from the patients in local language. A total of 18 patients were included in the study. 


\section{Exclusion criteria:}

Age $<18$ yrs and more than 70 years

Patients medically unfit for surgery

Patients not consenting to be a part of the study

Patients with history of previous surgery to ipsilateral axilla or arm.

Patients with clinically N2/N3 nodes

\section{Technique}

Reverse axillary mapping protocol: Patients included in the study were subjected to injection of methylene blue to a total volume of $1-1.5 \mathrm{ml}$ (a combination of intradermal and subcutaneous injection), given under general anaesthesia, prior to commencement of surgery . Intradermal injection was given using an insulin syringe, and subcutaneous injection using $2 \mathrm{ml}$ syringe with 26 gauze needle. All injections were given at the intermuscular groove in the medial aspect at the junction upper and middle third of the arm. Time from injection to the time of axillary dissection was noted. Careful and meticulous search for blue lymphatics were done at the start of axillary dissection to identify arm lymphatics. The position of the lymphatics/nodes were documented in relation to axillary vein, thoraco dorsal pedicle and intercostobrachial nerve. Blue nodes were dissected out and separately sent for histopathological analysis and routine axillary dissection was done to include levels 1,2 and 3 , limiting dissection to tissue below the axillary vein. Intraoperative assessment of the extent of axillary nodal disease was done to identify extensive disease and was correlated with post operative histopathology. Postoperative morbidity associated with the procedure was recorded. Patients were stratified based on the postoperative nodal status and degree of involvement of arm lymph nodes by metastatic disease assessed for each category (pNO,pN1,pN2,pN3).

Statistical Analysis:

Statistical analysis was performed using the SPSS Statistical Software (version 17).

\section{Results}

The median age of patients included was 51.44 years (34-60 years). Three of the 18 patients had underwent excision biopsy outside. Thirteen patients has clinically T2 lesion and 3 patients had clinically T1 lesions. Based on location of the primary tumour, upper outer quadrant (11) was the most common location, followed by upper inner quadrant (4), lower outer quadrant (1), lower inner quadrant(1) and central sector(1). Preoperative diagnosis of malignancy was available for all the patients (fine aspiration cytology (4), core needle biopsy (11) and slide review (3)). Intra ductal carcinoma NOS was the most common histology, one with neuroendocrine differentiation one with poorly differentiated carcinoma with neuro endocrine differentiation. Sixteen patients had grade 3 tumours, 2 patients with grade 2 tumours (Table 1). Modified radical mastectomy was performed in 15 of 18 patients, breast conservation surgery in 2 patients and axillary lymph node dissection(ALND) alone in one patient. Mean time from injection to axillary dissection was 41.07 minutes. Mean lymph node yield was 17.9 (range 11-32). Five patients had positive nodes in axilla on final histopathological assessment [pN1 in two ( one and two nodes positive ), pN3 in 3 patients(10,12 and 16 nodes positive)].All the three patients with $\mathrm{pN} 3$ could be identified intra operatively as suspicious of having high nodal involvement. Blue lymphatics(RAM lymphatics) were identified in 13 out of 18 patients (72.22\%) and blue lymphnodes were identified in 11 of 18 patients $(61.11 \%)$. Mean duration between the injection and start of axillary dissection was41.27 minutes. The blue nodes/lymphatics were most commonly located within a centimetre below the axillary vein, just lateral to thoracodorsal pedicle ( $9 / 11$ patients), anterior to the axillary vein(1) and just inferior to the vein (1).(Fig 1) Mean yield of blue lymph node was 1.83 (range 1-3). The size of the blue lymphnode was variable ranging from $4 \mathrm{~mm}$ to $1.7 \mathrm{~cm}$. Only one of the 11 patients in whom RAM lymph node was identified and removed, was positive for metastatic disease( had a high axillary nodal disease, pN3) (Fig 2) .Four of the 18 patients had minimal staining at the injection site, two patients complained of minimal pain at first review ( 2 weeks post surgery), one had a superficial epidermal loss and at the injection site

\section{Discussion}

Globally breast cancer is the most common cancer among women. The management of breast cancer has evolved over the years from most radical Halstead's Radical mastectomy through breast conservation surgery to sentinel node biopsy (SLNB), with a significant reduction in the morbidity associated with the procedure. The status of axilla is one of the most important predictors of disease outcome. Most important morbidity associated with breast cancer surgery is ipsilateral lymphedema. Lymphedema incidence has a direct relationship to the extent of axillary lymph node dissection(ALND), varying from $58.4 \%$ with radical mastectomy [12] to as low as $5 \%$ with sentinel lymph node techniques[13].Though Z0011 trial showed that SLNB alone without ALND was not inferior in terms of local recurrence and survival [14], still the standard 
practice is to perform an axillary dissection in women with positive SLNB.

Cadaveric studies tracing the lymphatic of upper limb, has shown that there are two pathways, a superficial and deep pathway, most of which drain into a single sentry node in the axilla[15]. Post ALND, most of these pathways become fibrosed, impairing lymphatic drainage of he upper limb, with opening of new lymphatics in some patients[16]. Lymphatics from the ipsilateral upper limb and breast in breast cancer patients draining into the axilla, were found to be different in $86.7 \%$ cases, in patients undergoing ALND[1].

A number of techniques have been described in literature for the identification of arm lymphatics in the axilla, using blue dye [4,6,8], radio isotope [9] and fluorescence[17]. Rate of Identification of arm lymphatics varies from $33.3 \%$ in initial studies to as high as $91 \%$ with use of radio isotopes, [8,9]. In our study, using methylene blue dye injection, at the start of surgery, blue lymphatics were identified in $72.2 \%$ while blue lymph nodes could be identified in $61.1 \%$. The pattern of distribution of arm lymphatics in axilla varies with respect to axillary vein . Bonetti et al described 5 common variations in the distribution of arm lymphatics in the axilla[6], with most of the lymphatics and lymph node being found within the region formed by inferior margin of axillary vein and the second intercostobrachial nerve and thoraco dorsal pedicle[4,5,11,17].In our study, $69.2 \%$ of the identified arm lymphatics was within one $\mathrm{cm}$ inferior to axillary vein, lateral to thoraco dorsal pedicle.RAM lymphatics were successfully identified in 10 of 13 patients with negative axilla, in $2 / 2$ patients with $\mathrm{pN} 1$ disease but only in $1 / 3$ patients with $\mathrm{pN} 3$ disease. This may be postulated to be secondary to lymphatic obstruction by extensive disease in axilla as described in literature $[9,18]$.

The mean time between injection and commencement of axillary dissection was 41.27 minutes for our patients, which was little longer than that described in literature [4,11]. The mean nodal yield from the axillary dissection was 17.9 (range 11- 32 nodes). 5 patients had positive nodes in axilla ( 2 had pN1 and 3 had pN3 status). During axillary dissection, intra operative findings suspicious of a high nodal yield was there in all the three patients with advanced nodal disease $(\mathrm{pN} 3)$, a findings not documented in previous series Intra operative assessment can help in identifying such patients who may be a high risk of harbouring metastasis in the RAM lymph nodes.

A total of 21 RAM node were identified and dissected out for histological analysis. The mean RAM node yield was 1.83 (range 1-3 nodes ) same as in previous studies[4,19]. Only one patient of 11 with RAM node identified, had metastatic disease in the RAM node (9.09\%) which is lesser when compared to rate described in literature (11-25\%) [10,19]. The positive RAM node was identified in pN3 axilla(high stage axillary disease). The same pattern had been described in various studies in literature, which have shown a positive relation between high nodal positivity and chance of arm lymphatics also being involved by the disease $[9,10,17,19,20]$ Schunemann Jr et al, found a statistically significant association between occurrence of metastasis in RAM nodes and high nodal disease $(\mathrm{P}<.0 .001)[19]$. Most studies thus suggest that performing RAM in patients with high nodal disease, is not oncologically sound $[10,11,17,19]$.

In our experience, even in patients without a preoperative suspicion of high nodal disease, intraoperative findings could correctly identify patients with high nodal disease (100\% in our series), and thus RAM node preservation may be avoided in them due to high possibility of involvement by metastatic disease. Ikeda et al further suggested that intra-operative fine needle aspiration cytology(FNAC) of the RAM node could be used to predict the their involvement. In their series, of the 7 patients with RAM node involvement, 5 were correctly identified as positive /suspicious on FNAC, while in two FNAC was inconclusive. They also found no discordance between the FNAC and final histopathology report. [17]

Use of blue dye injection (injection in the arm) has been found to be associated with few local morbidity, though no systemic reaction has been documented in literature. Nos et al, described persistent blue tattooing in the arm in more than 50\% of patients at a median follow up of 21 months, while Bedrosian et al., described a more milder reaction. $[4,10,11]$. In our series, there were no significant morbidity or mortality, only Four of the 18 patients had minimal staining at the injection site, at two weeks post surgery, while 2 patients complained of minimal pain, one had a superficial epidermal loss and at the injection site.

\section{Conclusion}

Reverse axillary mapping is a relatively new technique aimed at identifying lymphatic channel from the ipsilateral arm, draining into the axilla at the time of axillary management for patients with carcinoma breast(both sentinel node and nodal dissection). Identification rates have shown to be high in recent series, many have found a overlap between the ALND field /Sentinel node and RAM node. Its involvement by metastatic disease, hence the oncological safety in preserving them has been evaluated by small trials. Due to high rate of metastatic involvement in patients with high nodal disease, it is advisable to avoid preservation of RAM nodes, in patients with an intraoperative suspicion of high nodal disease. An alternative would be do perform an intraoperative FNAC from identified RAM node to rule out its metastatic involvement. Further larger studies needs to be performed to establish unequivocally the oncological safety of preservation of RAM nodes and also to evlaluate the long term outcomes in terms of lymphedema incidence and nodal recurrence, to 
establish this as a standard procedure.

\section{List of abbreviations}

RAM - Revrese Axillary Mapping

ALND - Axillary lymph node dissection

SLNB - Sentinel lymph node biopsy

FNAC - Fine needle aspiration cytology

\section{Declaration}

\section{Ethical consideration:}

The study was initially presented in the institute ethics committee and study initiated only after getting necessary approval.

\section{Consent for publication}

Obtained from the participating patients at the time of inclusion into the study

Availability of data and material - The datasets used and/or analysed during the current study are available from the corresponding author on reasonable request.

Competing interests: Nil

Funding : Nil

Author's Contribution:

Both authors contributed equally in the conception of idea, conduct of the study and analysis and writing up the results.

Nil

\section{Acknowledgement}

\section{References}

[1]. Britton TB, Solanki CK, Pinder SE, Mortimer PS, Peters AM, Purushotham AD. Lymphatic drainage pathways of the breast and the upper limb. Nucl Med Commun. 2009 Jun;30(6):427-30.

[2]. Masakuni Noguchi, Miki Yokoi, Yasuharu Nakano, Yukako Ohno and Takeo Kosaka (2011). Axillary Reverse Mapping in Breast Cancer, Radioisotopes - Applications in Bio-Medical Science, Prof. Nirmal Singh (Ed.),ISBN: 978-953-307-748-2,[Internet]. [cited 2015 Feb 8]. Available from: http://cdn.intechopen.com/pdfs-wm/23700.pdf

[3]. Thompson M, Korourian S, Henry-Tillman R, Adkins L, Mumford S, Westbrook KC, et al. Axillary reverse mapping (ARM): a new concept to identify and enhance lymphatic preservation. Ann Surg Oncol. 2007 Jun;14(6):1890-5.

[4]. Nos C, Lesieur B, Clough KB, Lecuru F. Blue dye injection in the arm in order to conserve the lymphatic drainage of the arm in breast cancer patients requiring an axillary dissection. Ann Surg Oncol. 2007 Sep;14(9):2490-6.

[5]. Noguchi M, Yokoi M, Nakano Y. Axillary reverse mapping with indocyanine fluorescence imaging in patients with breast cancer. J Surg Oncol. 2010 Mar 1;101(3):217-21.

[6]. Boneti C, Korourian S, Bland K, Cox K, Adkins LL, Henry-Tillman RS, et al. Axillary reverse mapping: mapping and preserving arm lymphatics may be important in preventing lymphedema during sentinel lymph node biopsy. J Am Coll Surg. 2008 May;206(5):1038-42; discussion 1042-4.

[7]. Kang S, Choi J, Jeon Y, Lee S, Bae Y. Preservation of lymphatic drainage from arm in breast cancer surgery: is it safe?. Cancer Res. 2009 Feb 19;69(2 Supplement):201-0.

[8]. Thompson M, Korourian S, Henry-Tillman R, Adkins L, Mumford S, Westbrook KC, et al. Axillary reverse mapping (ARM): a new concept to identify and enhance lymphatic preservation. Ann Surg Oncol. 2007 Jun;14(6):1890-5.

[9]. Nos C, Kaufmann G, Clough KB, Collignon M-A, Zerbib E, Cusumano P, et al. Combined axillary reverse mapping (ARM) technique for breast cancer patients requiring axillary dissection. Ann Surg Oncol. 2008 Sep;15(9):2550-5.

[10]. Ponzone R, Cont NT, Maggiorotto F, Cassina E, Mininanni P, Biglia N, et al. Extensive nodal disease may impair axillary reverse mapping in patients with breast cancer. J Clin Oncol Off J Am Soc Clin Oncol. 2009 Nov 20;27(33):5547-51.

[11]. Bedrosian I, Babiera GV, Mittendorf EA, Kuerer HM, Pantoja L, Hunt KK, et al. A phase I study to assess the feasibility and oncologic safety of axillary reverse mapping in breast cancer patients. Cancer. 2010 Jun 1;116(11):2543-8.

[12]. Deutsch M, Land S, Begovic M, Sharif S. The incidence of arm edema in women with breast cancer randomized on the National Surgical Adjuvant Breast and Bowel Project study B-04 to radical mastectomy versus total mastectomy and radiotherapy versus total mastectomy alone. Int J Radiat Oncol Biol Phys. 2008 Mar 15;70(4):1020-4.

[13]. Mansel RE, Fallowfield L, Kissin M, Goyal A, Newcombe RG, Dixon JM, et al. Randomized multicenter trial of sentinel node biopsy versus standard axillary treatment in operable breast cancer: the ALMANAC Trial. J Natl Cancer Inst. 2006 May 3;98(9):599-609.

[14]. Giuliano AE, Hunt KK, Ballman KV, Beitsch PD, Whitworth PW, Blumencranz PW, et al. Axillary dissection vs no axillary dissection in women with invasive breast cancer and sentinel node metastasis: a randomized clinical trial. JAMA. 2011 Feb 9;305(6):569-75.

[15]. Suami H, Taylor GI, Pan W-R. The lymphatic territories of the upper limb: anatomical study and clinical implications. Plast Reconstr Surg. 2007 May;119(6):1813-22.

[16]. Suami H, Pan W-R, Taylor GI. Changes in the lymph structure of the upper limb after axillary dissection: radiographic and anatomical study in a human cadaver. Plast Reconstr Surg. 2007 Sep 15;120(4):982-91.

[17]. Ikeda K, Ogawa Y, Komatsu H, Mori Y, Ishikawa A, Nakajima T, et al. Evaluation of the metastatic status of lymph nodes identified using axillary reverse mapping in breast cancer patients. World J Surg Oncol. 2012;10:233.

[18]. Tausch C, Baege A, Dietrich D, Vergin I, Heuer H, Heusler RH, et al. Can axillary reverse mapping avoid lymphedema in node 
positive breast cancer patients? Eur J Surg Oncol J Eur Soc Surg Oncol Br Assoc Surg Oncol. 2013 Aug;39(8):880-6

[19]. Schunemann E, Dória MT, Silvestre JBCH, Gasperin P, Cavalcanti TCS, Budel VM. Prospective study evaluating oncological safety of axillary reverse mapping. Ann Surg Oncol. 2014 Jul;21(7):2197-202.

[20]. Connor C, McGinness M, Mammen J, Ranallo L, Lafaver S, Klemp J, et al. Axillary reverse mapping: a prospective study in women with clinically node negative and node positive breast cancer. Ann Surg Oncol. 2013 Oct;20(10):3303-7.

\begin{tabular}{|l|l|l|l|l|l|l|l|l|l|}
\hline S.No & Age & Surgery & pT & $\begin{array}{l}\text { LN } \\
\text { yield }\end{array}$ & $\begin{array}{l}\text { Positive } \\
\text { LN }\end{array}$ & $\begin{array}{l}\text { RAM LN/ } \\
\text { Lymph }\end{array}$ & $\begin{array}{l}\text { RAM LN } \\
\text { yield }\end{array}$ & $\begin{array}{l}\text { RAM } \\
\text { positive }\end{array}$ \\
\hline 1 & 60 & MRM & 2 & 20 & 0 & Both & 2 & 0 & 4 \\
\hline 2 & 59 & MRM & 1 & 17 & 0 & Lymph & 0 & 0 & 44 \\
\hline 3 & 59 & MRM & 2 & 29 & 0 & Both & 3 & 0 & 45 \\
\hline 4 & 60 & MRM & 2 & 15 & 0 & Both & 2 & 0 & 46 \\
\hline 5 & 42 & MRM & 2 & 12 & 0 & Both & 2 & 0 & 42 \\
\hline 6 & 52 & MRM & 2 & 15 & 1 & lymph & 0 & 0 & 40 \\
\hline 7 & 52 & MRM & 2 & 24 & 0 & Both & 2 & 0 & 40 \\
\hline 8 & 44 & MRM & 2 & 18 & 0 & Both & 1 & 0 & 60 \\
\hline 9 & 60 & MRM & 1 & 13 & 0 & Both & 2 & 0 & 46 \\
\hline 10 & 44 & MRM & 2 & 11 & 0 & Both & 2 & 0 & 34 \\
\hline 11 & 45 & BCS & 1 & 12 & 0 & Both & 1 & 0 & 42 \\
\hline 12 & 53 & MRM & 2 & 25 & 15 & Both & 1 & 1 & 33 \\
\hline 13 & 53 & MRM & 2 & 12 & 12 & No & 0 & 0 & 35 \\
\hline 14 & 48 & ALND & 1 & 20 & 0 & No & 0 & 0 & 40 \\
\hline 15 & 52 & MRM & 2 & 17 & 0 & No & 0 & 0 & 48 \\
\hline 16 & 53 & MRM & 3 & 17 & 2 & Both & 3 & 0 & 38 \\
\hline 17 & 56 & MRM & 2 & 14 & 0 & No & 0 & 0 & 40 \\
\hline 18 & 34 & BCS & 1 & 32 & 10 & No & 0 & 0 & 35 \\
\hline
\end{tabular}

Table 1: Clinico-pathological characteristics of cohort.

Figure 1: Showing blue lymphatics in the axilla, which can be traced to identify the blue lymph node (RAM node)

Figure 2: Distribution of reverse axillary mapping lymph node with reference to axillary nodal yield ( RAM Reverse Axillary Mapping, LN - lymph node, + -positive for metastatic disease, pN - Denotes pathological staging (as per AJCC 7 th Edition)

(pT- Pathological T stage (as per AJCC 7th edition), MRM - modified radical mastectomy, BCS - breast conservation surgery), ALND -axillary lymph nodal dissection,LN - lymph node , Lymph- lymphatics, RAM - reverse axillary mapping

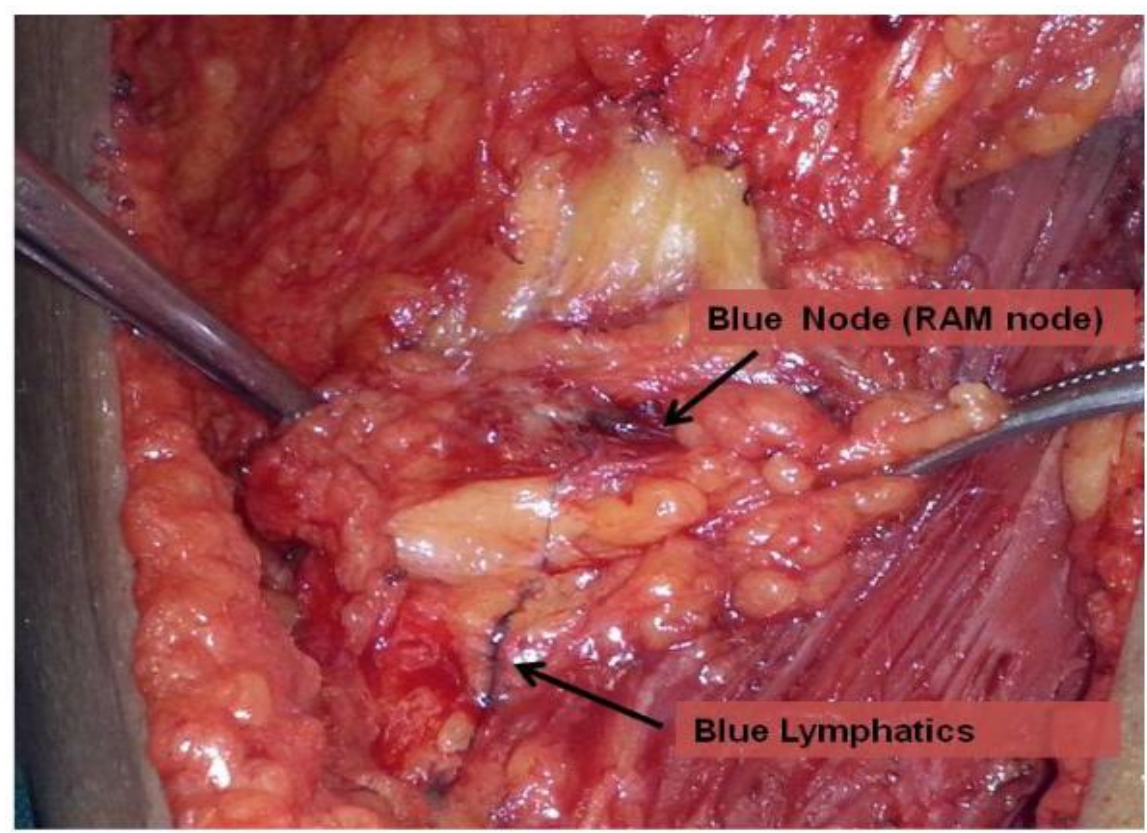




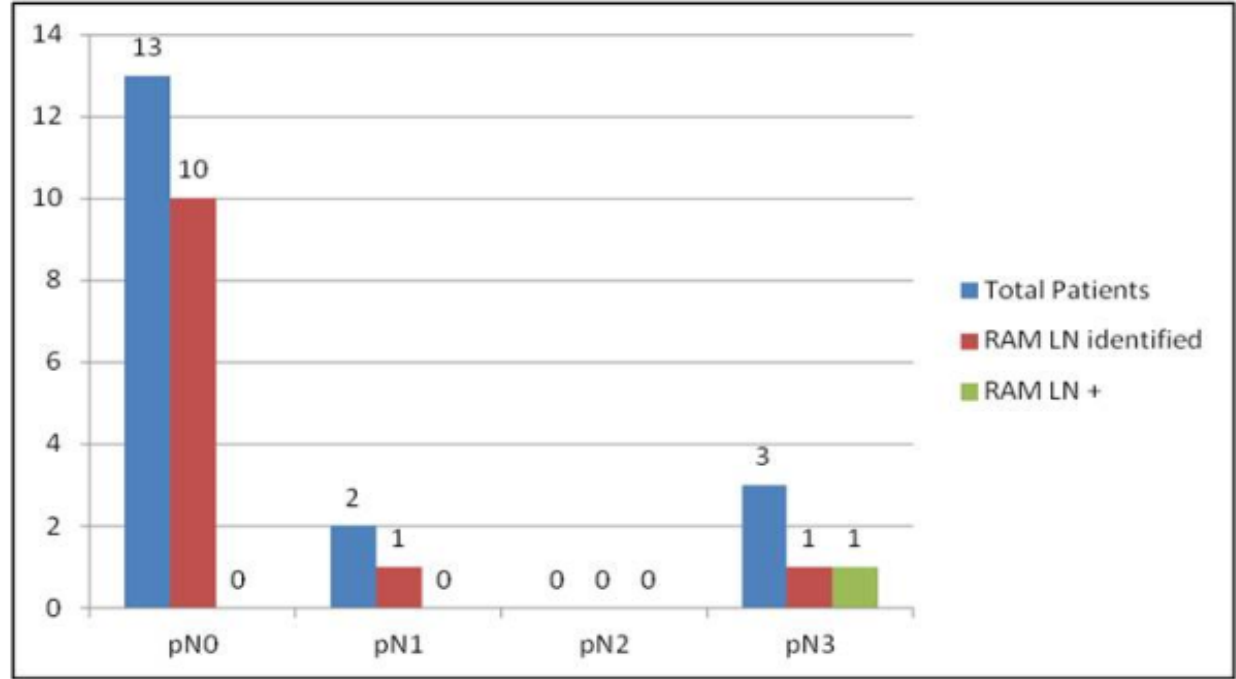

* Dr. Balasubramanian V. "“Reverse axillary mapping in early stage breast cancer Is it oncologically safe? A feasibity study"." IOSR Journal of Dental and Medical Sciences (IOSR-JDMS) 16.7 (2017): 110-15. 